\title{
Are There Chaotic Tilings?
}

\author{
Daniel Berend ${ }^{1,2 \star}$ and Charles Radin ${ }^{1 \star \star}$ \\ 1 Mathematics Department, University of Texas, Austin, TX 78712, USA \\ 2 Department of Mathematics and Computer Science, Ben-Gurion University, Beer-Sheva 84105, \\ Israel
}

Received May 11, 1992; in revised form July 28, 1992

\begin{abstract}
We develop a class of examples in the form of tiling dynamical systems for use as toy models in statistical mechanics, to analyze the possible existence of disordered crystals. We give the first such models which are disordered in the sense of having no discrete spectrum.
\end{abstract}

\section{Introduction}

Ten years ago, Ruelle published the paper "Do turbulent crystals exist?" [7], in which he suggested the existence of real materials which in thermal equilibrium at low temperature would be quite different microscopically from the usual periodic crystals; the suggested difference would be demonstrated by a diffraction spectrum which was absolutely continuous, even at zero temperature.

Ruelle's argument was based on a comparison of the usual classical statistical mechanical formalism with a typical dynamical system with $\mathbf{R}^{3}$ action $\left(\mathbf{R}^{3}\right.$ representing spatial translations), but without any detailed consideration of the structural role played by interacting particles in the former.

The present paper is motivated by the same problem, but with a different premise. We have chosen to concentrate on the special features which may be due to the role played by the interacting particles in statistical mechanics, with the aim to determine the qualitative low temperature features of generic classical statistical mechanical models with short range interactions. It is well known [7] that no such model has ever been proven to exhibit an ordered (crystalline) phase; presumably the reason is the difficulty in analyzing such models. To obtain results we first restrict attention to zero temperature, and then we distort the models to that of tiling dynamical systems (defined below), as is sometimes done in analyzing quasicrystals [8]. (Roughly speaking, in a tiling dynamical system the phase space 
consists of the tilings of Euclidean $n$-space, $E^{n}$, by copies of some finite set of shapes called tiles; intuitively, the way in which neighboring pairs of tiles need to fit together in a tiling replaces the short range interaction of mechanics.) It has been proven [4] that, generically, statistical mechanical models are uniquely ergodic with respect to spatial translations at zero temperature, and so we use this as an assumption in our models. In summary, the problem of the qualitative behavior of low temperature matter is here translated into: What is the range of qualitative behavior of uniquely ergodic tiling dynamical systems; in particular, do there exist such systems with absolutely continuous spectrum?

We introduce now some definitions. A tiling is a decomposition of $E^{n}$ into a union of "tiles" where:

(a) there is a fixed finite set $\mathscr{P}$ of "prototiles", which are homeomorphic images of the closed $n$-ball;

(b) each tile is an isometric copy of some prototile,

(c) the interiors of the tiles do not overlap,

(d) the isometries in (b) are restricted to some fixed subgroup $G$ of the full isometry group of $E^{n}$.

We endow the space $V(\mathscr{P})$ (assumed nonempty) of all tilings by some given set $\mathscr{P}$ of prototiles with a topology. Intuitively, tilings should be close if they differ only slightly inside some large bounded region. A finite set of nonoverlapping tiles will be called a swatch. We define a countable base for the topology on $V(\mathscr{P})$, using some countable dense subset $G^{\prime}$ of the topological subgroup $G$ (usually $\mathbf{Z}^{n}$ or $\mathbf{R}^{n}$ ) of the isometry group of $E^{n}$, as follows. Given a positive integer $k$, a set of positive rationals $\left\{r_{j}\right\}_{1}^{k}$, and a swatch of tiles $\left\{g_{j}^{\prime}\left(P_{j}^{\prime}\right)\right\}_{1}^{k}$ (where $g_{j}^{\prime} \in G^{\prime}, P_{j}^{\prime} \in \mathscr{P}$ ), we define the open set consisting of all tilings containing a swatch $\left\{g_{j}\left(P_{j}\right)\right\}_{1}^{k}$ such that $h\left[g_{j}\left(P_{j}\right)\right.$, $\left.g_{j}^{\prime}\left(P_{j}^{\prime}\right)\right]<r_{j}$ for all $j \leqq k$, where $h$ is the Hausdorff metric on compact sets. We note that the space $V(\mathscr{P})$, of tilings from a given prototile set $\mathscr{P}$, is compact and metrizable, and $G$ acts continuously on $V(\mathscr{P})[6]$.

Now consider any (one-dimensional) subshift $(X, T)$ over a finite alphabet $\mathscr{A}$, with lattice translation denoted by $T$. We will need to refer on occasion to the cylinder sets $\mathscr{C}_{a} \equiv\left\{x \in X: x_{0}=a\right\}, a \in \mathscr{A}$. Then, given a positive realvalued function $f$ on the alphabet $\mathscr{A}$, we associate with this subshift $(X, T)$, which is a discrete dynamical system, the continuous dynamical system $\left(X_{f}, T_{f}\right)$ defined as follows. $X_{f}$ is the subset of all tilings of $\mathbf{R}$ by translations of closed intervals $[0, a]$, where $a$ is in the range of $f$, and the sequence of intervals $I$, of length $|I|$, is such that any corresponding sequence of letters $f^{-1}(|I|) \in \mathscr{A}$ is in $X . X_{f}$ is easily seen to be a closed subset of the space of all tilings by such intervals, and invariant under translations, which are denoted by $\left\{T_{f}^{t}: t \in \mathbf{R}\right\}$. (We use below the obvious equivalence of $\left(X_{f}, T_{f}\right)$ with the classical construct of a flow under a function.)

We now specialize to the case where $(X, T)$ is the substitution dynamical system determined by the substitution $\xi$ :

$$
\xi(0)=0101, \quad \xi(1)=1110 .
$$

We note that $(X, T)$ is uniquely ergodic [3], which easily implies that $\left(X_{f}, T_{f}\right)$ is also uniquely ergodic for any $f$. 


\section{Results}

Our first result is the following

Lemma. Let $(X, T)$ be the substitution dynamical system defined by the substitution $\xi$ given by (1). If $f(0)$ and $f(1)$ are rationally independent, then the associated continuous system $\left(X_{f}, T_{f}\right)$ has no discrete spectrum.

Proof. Suppose we have an eigenfunction $g$ of the family $T_{f}^{t}$ :

$$
T_{f}^{t} g(x, s)=e^{2 \pi i \lambda t} g(x, s) .
$$

Let $u$ be the function on $X$ defined as the restriction of $g$ to some height $s_{0}$, $0 \leqq s_{0}<\min \{f(0), f(1)\}$ (where (2) holds for all $t$ almost everywhere):

$$
u(x)=g\left(x, s_{0}\right), \quad x \in X .
$$

In view of (2) we have:

$$
T u(x)= \begin{cases}e^{2 \pi i \lambda f(0)} u(x), & x \in \mathscr{C}_{0} \\ e^{2 \pi i \lambda f(1)} u(x), & x \in \mathscr{C}_{1} .\end{cases}
$$

Defining a sequence of functions $f_{n}: X \rightarrow \mathbf{R}_{+}, n \geqq 1$, by

$$
f_{n}(x)=\sum_{k=0}^{4^{n}-1} f\left(\left[T^{k} x\right]_{0}\right),
$$

we have:

$$
T^{4^{n}} u=e^{2 \pi i \lambda f_{n}} u .
$$

As in the proof of Theorem 1 in [1], this implies that $e^{2 \pi i \lambda f_{n}} \stackrel{L^{2}}{\longrightarrow} 1$, or, equivalently,

$$
\lambda f_{n} \stackrel{L^{2}}{\longrightarrow} 0(\bmod 1)
$$

(where $\stackrel{L^{2}}{\longrightarrow}$ denotes convergence in $L^{2}$ norm). Again as in [1] we can find sets $A_{n} \subseteq X$ whose measures are bounded away from 0 such that

$$
f_{n}(x)=\frac{4^{n}-1}{3} f(0)+\frac{2 \cdot 4^{n}+1}{3} f(1), \quad x \in A_{n} .
$$

From (3) and (4) it follows that:

$$
4^{n} \lambda \frac{f(0)+2 f(1)}{3} \rightarrow \lambda \frac{f(0)-f(1)}{3}(\bmod 1) .
$$

In particular, the right-hand side is invariant under multiplication by 4 modulo 1 :

$$
4 \lambda \frac{f(0)-f(1)}{3}=\lambda \frac{f(0)-f(1)}{3}(\bmod 1) .
$$

Consequently, $\lambda f(1)=\lambda f(0)+l$ for some $l \in \mathbf{Z}$. Again by (5), $3 \lambda f(0)+2 l$ is a (2-adic) rational, so both $\lambda f(0)$ and $\lambda f(1)$ are rationals. As $f(0)$ and $f(1)$ are rationally independent, this implies $\lambda=0$. This means in turn that $u$ is a $T$ invariant function. As $T$ is ergodic, $u$ is constant almost everywhere. Also, since $\lambda=0$ the function $g$ is constant as a function of $s$ for each fixed $x$. Thus, $g$ is constant in both variables. 
We now need to make use of a technique of Mozes [2], who showed how, given any two substitution dynamical systems (satisfying some mild conditions), one can construct a (large) set of prototiles in $E^{2}$, each of which is a unit square with small bumps and dents on its edges, all centered at the origin in the plane and with edges aligned, such that the associated discrete dynamical system (with $\mathbf{Z}^{2}$ action) is uniquely ergodic, and is measure-theoretically isomorphic to the product of the two given substitution dynamical systems. If the systems are $\left(X_{1}, T_{1}\right)$ and $\left(X_{2}, T_{2}\right)$, with alphabets $\mathscr{A}_{1}$ and $\mathscr{A}_{2}$, the product is $\left(X_{1} \times X_{2}, \mathbf{T}\right)$, where $\mathbf{T}=\left(T_{1}^{j} \times T_{2}^{k}\right)_{j, k=-\infty}^{\infty}, T_{1}^{j} \times T_{2}^{k}: \quad\left(x_{1}, x_{2}\right) \rightarrow\left(T_{1}^{j} x_{1}, T_{2}^{k} x_{2}\right)$. The isomorphism is a simple one-to-one correspondence between the tilings and the elements of $X_{1} \times X_{2}$; there is a many-to-one correspondence between the prototiles and pairs $(a, b) \in \mathscr{A}_{1} \times \mathscr{A}_{2}$, and each tiling, which is a two-dimensional array of (essentially) unit square tiles, is naturally identifiable with the corresponding element of $X_{1} \times X_{2}$. (Just assume that each tile has inscribed on it a pair $(a, b) \in \mathscr{A}_{1} \times \mathscr{A}_{2}$ as above, so that each tiling is associated in the obvious way with the unique element of $X_{1} \times X_{2}$.)

We now modify the above to adapt it to continuous tilings. We begin by using the substitution dynamical system $(X, T)$ defined by (1) for both factors in the construction of Mozes. Next we modify the shapes of the "square" prototiles that the construction produces, by stretching each one associated with the pair $(a, b) \in$ $\{0,1\} \times\{0,1\}$ to a "rectangle" with horizontal edges $f(a)$ and vertical edges $f(b)$, where $f(0)$ and $f(1)$ are fixed and rationally independent. The lemma then implies that the continuous tiling system thus produced is weakly mixing. Let $\left(V(\mathscr{P}), \mathbf{R}^{2}\right)$ be this tiling system.

Theorem. $\left(V(\mathscr{P}), \mathbf{R}^{2}\right)$ is a continuous, uniquely ergodic dynamical system which is weakly mixing; that is, it has no discrete spectrum.

\section{Closing Remarks}

Note that we describe the disorder of our examples by means of invariant probability measures. It is essential for our purposes that there is no choice involved with these measures; they are uniquely defined by the prototile sets themselves. If one could choose an invariant measure, there would be no depth to the subject: one could very easily find a prototile set with associated dynamics which was very wild. For example, the one-dimensional continuous tiling system, with prototile set consisting of two intervals of incommensurate lengths, is strongly mixing if one chooses an appropriate measure; just use the method of the introduction, applying the flow under a function to the "Bernoulli" shift, namely $X=\{0,1\}^{\mathbf{Z}}$, equipped with the product measure $\mu_{B}$ for which $\mu_{B}\left(\mathscr{C}_{0}\right)=\mu_{B}\left(\mathscr{C}_{1}\right)=1 / 2$. (Physically, there is no surprise that a noninteracting particle system was a unique zero temperature Gibbs state which has absolutely continuous spectrum. The result is uninteresting because it does not represent a single structure, but a highly degenerate average over many structures.) On the other hand, it is a major unsolved problem to determine whether or not there is a uniquely ergodic tiling dynamical system (discrete or continuous) which has purely absolutely continuous spectrum [5], which we would call "chaotic", and which explains the title of this paper. The method in [5] for producing a weakly mixing discrete tiling system, which uses a tower over the substitution system $(X, T)$ defined by (1) for each factor in the 
construction of Mozes, cannot produce a strongly mixing (discrete) system since the factors, being themselves substitution systems, cannot be strongly mixing $[1,3]$. It is unclear to us whether or not this new method can produce a strongly mixing (continuous) system.

\section{References}

1. Dekking, F.M., Keane, M.: Mixing properties of substitutions. Zeit. Wahr. 42, 23-33 (1978)

2. Mozes, S.: Tilings, substitution systems and dynamical systems generated by them. J. d'Analyse Math. 53, 139-186 (1989)

3. Queffélec, M.: Substitution Dynamical Systems - Spectral Analysis. Berlin: Springer 1987

4. Radin, C.: Global order from local sources. Bull. Am. Math. Soc. 25, 335-364 (1991)

5. Radin, C.: Disordered ground states of classical lattice models. Revs. Math. Phys. 3, 125-135 (1991)

6. Radin, C., Wolf, M.: Space tilings and local isomorphism. Geometriae Dedicata 42, 355-360 (1992)

7. Ruelle, D.: Do turbulent crystals exist?. Physica 113A, 619-623 (1982)

8. Steinhardt, P., Ostlund, S.: The Physics of Quasicrystals. Singapore: World Scientific 1987

Communicated by J.-P. Eckmann 
